\title{
Scoping review identifies significant number of knowledge translation theories, models and frameworks with limited use
}

\author{
Lisa Strifler, Roberta Cardoso, Jessie McGowan, Elise Cogo, Vera \\ Nincic, Paul A. Khan, Alistair Scott, Marco Ghassemi, Heather \\ MacDonald, Yonda Lai, Victoria Treister, Andrea C. Tricco, and \\ Sharon E. Straus
}

Version Post-print/Accepted Manuscript

Citation Strifler L, Cardoso R, McGowan J, Cogo E, Nincic V, Khan PA, Scott (published version) A,Ghassemi M, MacDonald H, Lai Y, Treister V, Tricco AC, Straus SE, Scoping review identifies significant number of knowledge translation theories, models and frameworks with limited use, Journal of Clinical Epidemiology (2018), doi: 10.1016/j.jclinepi.2018.04.008.

Copyright/License

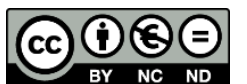
This work is licensed under the Creative Commons Attribution-NonCommercial-NoDerivatives 4.0 International License. To view a copy of this license, visit http://creativecommons.org/licenses/by-nc-nd/4.0/.

How to cite TSpace items

Always cite the published version, so the author(s) will receive recognition through services that track citation counts, e.g. Scopus. If you need to cite the page number of the author manuscript from TSpace because you cannot access the published version, then cite the TSpace version in addition to the published version using the permanent URI (handle) found on the record page.

This article was made openly accessible by $U$ of $T$ Faculty. Please tell us how this access benefits you. Your story matters. 


\section{Accepted Manuscript}

Scoping review identifies significant number of knowledge translation theories, models and frameworks with limited use

Lisa Strifler, $\mathrm{PhD}$ (cand), Roberta Cardoso, PhD, Jessie McGowan, PhD, Elise Cogo, ND, Vera Nincic, PhD, Paul A. Khan, PhD, Alistair Scott, BSc, Marco Ghassemi, MSc, Heather MacDonald, MSc, Yonda Lai, MN, Victoria Treister, MPH, Andrea C. Tricco, $\mathrm{PhD}$, Sharon E. Straus, MD

PII: S0895-4356(17)31417-8

DOI: 10.1016/j.jclinepi.2018.04.008

Reference: JCE 9635

To appear in: Journal of Clinical Epidemiology

Received Date: 24 December 2017

Revised Date: 27 March 2018

Accepted Date: 6 April 2018

Please cite this article as: Strifler L, Cardoso R, McGowan J, Cogo E, Nincic V, Khan PA, Scott A, Ghassemi M, MacDonald H, Lai Y, Treister V, Tricco AC, Straus SE, Scoping review identifies significant number of knowledge translation theories, models and frameworks with limited use, Journal of Clinical Epidemiology (2018), doi: 10.1016/j.jclinepi.2018.04.008.

This is a PDF file of an unedited manuscript that has been accepted for publication. As a service to our customers we are providing this early version of the manuscript. The manuscript will undergo copyediting, typesetting, and review of the resulting proof before it is published in its final form. Please note that during the production process errors may be discovered which could affect the content, and all legal disclaimers that apply to the journal pertain. 
1 Scoping review identifies significant number of knowledge translation theories, models and

2 frameworks with limited use

3

$4 \quad$ Lisa Strifler ${ }^{1,2} \mathrm{PhD}$ (cand) $\quad$ Email: striflerl@ smh.ca

5 Roberta Cardoso ${ }^{1} \mathrm{PhD} \quad$ Email: cardosor@smh.ca

6 Jessie McGowan ${ }^{3} \mathrm{PhD} \quad$ Email: jmcgowan@uottawa.ca

7 Elise Cogo ${ }^{1} \mathrm{ND} \quad$ Email: elisec1234@gmail.com

$8 \quad$ Vera Nincic ${ }^{1} \mathrm{PhD} \quad$ Email: nincicv@smh.ca

9 Paul A. Khan ${ }^{1} \mathrm{PhD} \quad$ Email: khanp@smh.ca

$10 \quad$ Alistair Scott ${ }^{1}$ BSc $\quad$ Email: scotta@smh.ca

11 Marco Ghassemi ${ }^{1}$ MSc $\quad$ Email: ghassemim@smh.ca

12 Heather MacDonald ${ }^{1}$ MSc $\quad$ Email: macdonaldh@smh.ca

13 Yonda Lai ${ }^{1} \mathrm{MN} \quad$ Email: laiy@smh.ca

14 Victoria Treister ${ }^{1}$ MPH $\quad$ Email: treisterv@smh.ca

15 Andrea C. Tricco ${ }^{1,4} \mathrm{PhD} \quad$ Email: triccoa@ smh.ca

16 Sharon E. Straus ${ }^{* 1,5}$ MD $\quad$ Email: sharon.straus@utoronto.ca

$18{ }^{1}$ Li Ka Shing Knowledge Institute, St. Michael's Hospital, 209 Victoria Street, East Building,

19 Toronto, Ontario, M5B 1W8, Canada.

$20 \quad{ }^{2}$ Institute of Health Policy Management \& Evaluation, University of Toronto, 4th Floor, 155

21 College Street, Toronto, Ontario, M5T 3M6, Canada.

$22{ }^{3}$ School of Epidemiology, Public Health and Preventive Medicine, University of Ottawa, 600

23 Peter Morand Crescent, Ottawa, Ontario, K1G 5Z3, Canada. 
$24{ }^{4}$ Epidemiology Division, Dalla Lana School of Public Health, University of Toronto, 6th Floor, 25155 College Street, Toronto, Ontario, M5T 3M7, Canada.

$26{ }^{5}$ Department of Geriatric Medicine, University of Toronto, 27 King's College Circle, Toronto, 27 Ontario, M5S 1A1, Canada.

28

$29{ }^{*}$ Corresponding Author

30 Dr. Sharon E. Straus

31 Knowledge Translation Program, Li Ka Shing Knowledge Institute, St. Michael's Hospital

32209 Victoria Street, East Building, Room 716, Toronto, Ontario, M5B 1W8, Canada

33 Telephone: 1-416-864-3068, Fax: 416-864-5805, E-mail: sharon.straus@utoronto.ca 


\section{ABSTRACT}

35 Objective: To conduct a scoping review of knowledge translation (KT) theories, models and

36 frameworks that have been used to guide dissemination or implementation of evidence-based

37 interventions targeted to prevention and/or management of cancer or other chronic diseases.

38 Study design and setting: We used a comprehensive multistage search process from 2000-2016,

39 which included traditional bibliographic database searching, searching using names of theories,

40 models and frameworks, and cited reference searching. Two reviewers independently screened

41 the literature and abstracted data.

42 Results: We found 596 studies reporting on the use of 159 KT theories, models or frameworks. A 43 majority $(87 \%)$ of the identified theories, models or frameworks were used in five or fewer

44 studies, with $60 \%$ used once. The theories, models and frameworks were most commonly used to

45 inform planning/design, implementation and evaluation activities, and least commonly used to

46 inform dissemination and sustainability/scalability activities. Twenty-six were used across the full

47 implementation spectrum (from planning/design to sustainability/scalability) either within or

48 across studies. All were used for at least individual-level behavior change, while $48 \%$ were used

49 for organization-level, $33 \%$ for community-level and $17 \%$ for system-level change.

50 Conclusion: We found a significant number of KT theories, models and frameworks with a

51 limited evidence base describing their use.

53 Keywords (max 6): Knowledge synthesis, Knowledge translation, Implementation, Theory,

54 Model, Framework

55 Running title: Scoping review of knowledge translation theories, models and frameworks

56 Word count: 200 (Abstract - 200 max), 4725 (Main text - 3000 - 5000), 2 Figures, 2 Tables, 1

57 Supplementary File 
What is new?

\section{$59 \quad$ Key findings}

60 - We employed a new, iterative approach called the BeHEMoTh (Behavior of interest; Health

61 context; Exclusions; Models or Theories) framework to inform our search protocol and to

62 build a list of names of knowledge translation (KT) theories, models and frameworks.

63 - Our scoping review identified 596 studies reporting on the use of 159 KT theories, models or

64 frameworks. A majority of the theories, models and frameworks were used in less than $1 \%$

65 (i.e., five or fewer) of the included studies, with many used once.

\section{What this adds to what was known?}

- To our knowledge, this is the first comprehensive review of KT theories, models and

68 frameworks that have been used to guide dissemination or implementation of evidence-based

69 interventions targeted to prevention and/or management of cancer or other types of chronic

$70 \quad$ diseases.

71 What is the implication and what should change now?

72 - We found a significant number of KT theories, models and frameworks with a limited

73 evidence base describing their use in practice. Our results suggest that a decision support tool

74 to help end users identify an appropriate theory, model or framework to inform their KT

75 activities would be beneficial. 


\section{INTRODUCTION}

Knowledge translation (KT) theories, models and frameworks are beneficial when implementing and sustaining evidence-based chronic disease and cancer control practices and policies. In particular, they may improve the likelihood of successful implementation and sustainability $[1,2]$. Yet studies have shown that researchers fail to use them or may not use them appropriately $[3,4]$.

81 Given that theories, models and frameworks serve different purposes, using a taxonomy may help

82 to facilitate their uses in practice and research [5]. For example, Nilsen's 2015 taxonomy [5]

83 describes KT theories as useful for understanding or explaining aspects of implementation (i.e.,

84 the mechanism of change). Examples include Rogers's Theory of Diffusion [6], Bandura's Social

85 Cognitive Theory [7], and May's Normalization Process Theory [8]. Nilsen's taxonomy describes

86 KT models as useful for guiding the steps or process of implementation. Graham et al.'s

87 Knowledge-to-Action Framework [9] and Meyers et al.'s Quality Implementation Framework

88 [10] are examples of such models. Finally, KT frameworks are described as useful for understanding or explaining influences on implementation outcomes or for evaluating implementation efforts [5]. Michie et al.'s Theoretical Domains Framework [11], Damschroder et al.’s CFIR (Consolidated Framework for Implementation Research) [12], and Glasgow et al.'s RE-AIM (Reach Effectiveness Adoption Implementation Maintenance) [13] framework are examples that meet this definition.

There are over 100 identified KT theories, models and frameworks to choose from (e.g., Nilsen, 2015 [5]; Tabak et al., 2012 [14]; Straus et al., 2013 [15]). Yet few studies have methodically examined their use in practice or research [16]. A recent citation network analysis of $63 \mathrm{KT}$ theories, models and frameworks found that while some were highly cited, most were rarely cited 
in the literature from 1985 to 2012 [17]. A rigorous knowledge synthesis is required to identify which theories, models and frameworks have been used to inform the spectrum of KT activities across the different levels of behavior change and to provide guidance on what theories, models and frameworks might be useful for implementation researchers and practitioners. To fill this gap, and in response to a proposal request from the Canadian Partnership Against Cancer for a rapid review, we conducted a scoping review of theories, models and frameworks used in KT to guide dissemination or implementation of evidence-based interventions targeted to prevention or management of cancer or other chronic diseases.

\section{METHODS}

\subsection{Protocol}

110 Our protocol was developed using the scoping review methods by Arksey and O’Malley (2005)

111 [18] and outlined in the Joanna Briggs Institute Reviewers' Manual [19]. We defined KT as "a

112 dynamic and iterative process that includes synthesis, dissemination, exchange and ethically-

113 sound application of knowledge" [20]. As such, we considered KT broadly to include both

114 implementation practice (i.e., implementing research evidence into practice) and implementation

115 science, which we defined as "the systematic study of specified activities designed to put into

116 practice activities or programs of known dimensions" [21]. The $\mathrm{P}$ (patient, problem or population)

117 I (intervention) $\mathrm{C}$ (comparison, control or comparator) $\mathrm{O}$ (outcome) and eligibility criteria were

118 specified a priori with input from our knowledge users (LZ, JK) at the Canadian Partnership

119 Against Cancer (a federally-funded organization that is the steward of the Canadian Strategy for

120 Cancer Control). We used an integrated KT approach to our scoping review, defined as "an

121 approach to doing research that applies the principles of KT to the entire research process [by] 
involving knowledge users as equal partners alongside researchers" [22]. Specifically, our knowledge users were engaged at all phases including the protocol construction, literature search, screening, data abstraction, synthesis, and dissemination. We chose to follow the PRISMA

125 (Preferred Reporting Items for Systematic Reviews and Meta-Analyses) statement [23] for reporting our results, since a reporting tool for scoping reviews does not yet exist.

\subsection{Eligibility criteria}

129 All theories, models and frameworks relevant to evidence dissemination or implementation,

130 whether developed in health or other fields (e.g., business, social sciences) were eligible for

131 inclusion. Specifically, we searched for articles reporting on dissemination or implementation of

132 any KT intervention (a) designed to change behavior at the level of the individual, organization,

133 community, or system, and (b) targeted to prevention or management of cancer or other types of

134 chronic disease (including chronic mental illness such as mood disorders including depression

135 and anxiety, schizophrenia, dementia, drug addiction) and related risk factors (such as smoking,

136 exercise, alcohol, diet, hypertension, obesity, tobacco and other risk factor modification), in all

137 relevant healthcare and community settings, and for all ages. We included all study designs,

138 including experimental, quasi-experimental, observational and qualitative. Articles were excluded

139 if they mentioned a KT theory, model or framework (e.g., in the introduction or discussion

140 sections) without reporting on using it to guide dissemination or implementation of a KT

141 intervention. We also excluded articles focused on describing or discussing the development of a

142 KT theory, model or framework. To increase feasibility, we excluded non-English articles, those

143 published before the year 2000, book chapters, dissertations and reviews. Conference abstracts 
144 and study protocols were only considered for inclusion if the study results (preliminary or full)

145 had been published.

146

\subsection{Information sources and search strategy}

148 Theory is a difficult subject to search as the language used to describe theories, models and

149 frameworks is inconsistent, which creates a challenge for the searcher. To develop a more

150 systematic approach to searching this subject area, we employed a new, iterative approach called

151 the BeHEMoTh (Behavior of interest; Health context; Exclusions; Models or Theories)

152 framework [24] to inform our search protocol and to build a list of names of KT theories, models

153 and frameworks. This approach was specifically developed to provide a template and process to

154 identify theories, models and frameworks for developing and testing complex interventions for

155 different types of systematic reviews. Before we began the search, the team prepared the question

156 using the BeHEMoTh question formulation.

157

158 The first stage of the search protocol included searching bibliographic databases for 'Behavior of interest' and 'Health context' and then consolidating the leads from this approach using a

160 systematic search process to build a list of names of KT theories, models and frameworks. We

161 operationalized the 'Behavior of interest' as any KT intervention designed to change behavior at

162 the level of individual, organization, community, or system. We operationalized the 'Health

163 context' as the prevention or management of cancer or other chronic diseases. 'Exclusions' were

164 non-theoretical/technical models such as statistical models. Using BeHEMoTH, we

165 operationalized theory in a search string as 'model* or theor* or concept* or framework*'. We

166 developed the search strategy by combining subject headings and text words for the 'Behavior of 
interest' AND 'Health context' AND the theory search string. We ran the finalized search strategy in MEDLINE, EMBASE, and PsycINFO on July 5, 2016 and included limits for publication language (English only) and publication date (2000 to 2016) to increase feasibility. The MEDLINE version of the search strategy was peer reviewed independently by an information specialist (EC) using the Peer Review of Electronic Search Strategies (PRESS) criteria [25] and modified, as needed. The MEDLINE search strategy was then adapted to the other databases. The final MEDLINE search strategy is found in Appendix A (Supplementary file).

In the second stage of the search protocol, we compiled a list of relevant named theories, models and frameworks identified during the first stage (i.e., extracted during title and abstract screening). We supplemented this list with additional KT theories, models and frameworks identified from existing published knowledge syntheses [5, 14, 15], for a total of 305 names. The full list of KT theories, models and frameworks is found in Appendix B (Supplementary file).

181 The third stage of the search included a 'named item' search based on identified theories, models and frameworks found in stage two combined with subject headings and text words for 'Health context'. This search was conducted in MEDLINE, EMBASE, and PsycINFO on August 18, 2016 and January 6, 2017, and was limited by publication language (English only) and publication date (2000 to 2016).

187 The fourth stage of the search protocol was a cited reference search using the Web of Science 188 database. The first 285 citations of screened and potentially relevant articles identified in the 189 previous stages, were entered into Excel. Using the RANDBETWEEN function in Excel, we 
created a randomized sample of $15 \%$. Within the cited references, a sub search was used to identify 'Behavior of interest', to limit citing articles to citations featuring the KT concepts. The search was run on January 10, 2017 and was limited to article type (primary studies) and publication date (2000 to 2016).

In addition, we used the Canadian Agency for Drugs and Technologies in Health (CADTH) Grey Matters approach to guide our search of the grey literature [26]. Specifically, we scanned national and international websites that were identified by a KT expert (SES) and an experienced research librarian (JM). The list of websites is found in Appendix C (Supplementary file). Finally, to ensure literature saturation, we searched for additional articles by screening the reference lists of relevant systematic reviews identified during the first three stages of screening. We combined the results from all stages of searching and removed duplicate citations.

\subsection{Study selection process}

204 Two reviewers independently screened titles and abstracts (level 1) and full-text articles (level 2)

205 using online systematic review software Synthesi.sr [27]. Prior to screening, all reviewers

206 completed two separate calibration exercises at each level to ensure reliability. Inter-rater agreement was calculated using percent agreement (>75\%) across all reviewers. We achieved acceptable agreement after two random samples of 50 articles at level 1, and two sets of 20 articles at level 2. Discrepancies between reviewers were resolved by consensus, or by a third

210 reviewer (LS, SES).

\subsection{Data items and abstraction process}


213 Two reviewers independently abstracted data from each article. Prior to data abstraction, all

214 reviewers completed two calibration exercises on a total of 10 articles to ensure team agreement

215 through consensus. Data abstraction items included study characteristics (e.g., year, geographic

216 region, context, funding source), KT theory, model or framework characteristics (e.g., name,

217 reference, description of how it was used and at which stage of KT practice), and KT intervention

218 characteristics (e.g., study design, description of intervention and comparator groups, setting,

219 target, level of behavior change, stage of care, outcomes), as well as the evidence-based clinical

220 intervention. The stages of KT practice were categorized as dissemination, planning/design,

221 implementation, evaluation, and sustainability/scalability based on Graham et al.'s Knowledge-to-

222 Action process model [9]. For example, the planning/design stage included identifying barriers

223 and facilitators to behavior change and assessing organizational readiness to change, and the

224 implementation stage included selecting, tailoring, implementing, and monitoring KT interventions. The KT intervention setting was defined as community (e.g., schools, community organizations), outpatient clinic, hospital, home, government, or long-term care. The KT intervention target was abstracted using the Effective Practice and Organisation of Care (EPOC) taxonomy and categorized as patient/general public interventions, healthcare professional interventions, organizational interventions, financial interventions, and regulatory interventions

230 [28]. For example, KT interventions targeting patients, family members, and/or the general public 231 directly were classified as patients/public. The level of behavior change was abstracted using a

232 broad socio-ecological framework of individual, organizational, community, and system [29]. For 233 example, KT interventions focused on changing knowledge, attitudes, and/or behaviors at the 234 level of the individual were classified as individual level change. The stage of care was abstracted 235 using the care continuum categories of prevention, screening, diagnosis, clinical care/treatment, 
prognosis, survivorship, and palliative/end-of-life care. Outcomes were abstracted and then coded

using the Cochrane taxonomy of relevant outcomes [30]. To optimize feasibility, we did not

contact study authors for clarity on the theories, models or frameworks used, or to obtain

information missing from the included study.

\subsection{Methodological quality appraisal}

242 We did not appraise the quality of the studies included in our scoping review, which is consistent

243 with the Joanna Briggs Institute Reviewers' Manual [19] as well as scoping reviews on clinical

244 topics [31].

\subsection{Synthesis}

247 We summarized the included studies based on study characteristics, KT theory, model or

248 framework characteristics, and KT intervention characteristics as reported in the individual

249 studies. Based on input from our knowledge users at the Canadian Partnership Against Cancer,

250 subgroup analyses were performed to explore the context (cancer, chronic disease, both), stage of

251 care (prevention, screening, diagnosis, treatment, prognosis, survivorship, palliative care), and

252 level of behavior change (individual, organization, community, system).

\section{3. RESULTS}

\subsection{Literature search}

256 We identified 1664 citations from our first search; 1989 citations from our named search; 235

257 citations from our cited reference search; 977 citations from our grey literature search of 24

258 websites; and 319 citations from checking the reference lists of 31 systematic reviews. After 
removing duplicates, we screened 4598 citations at the title and abstract level and 1899 articles at

260 the full-text level. Overall, we included 596 studies and 47 companion reports in our scoping

261 review (Figure 1). Of the 596 includes, 122 articles were from our grey literature search and all

262 articles were published. The full citation list for the 596 included articles and 47 companion

263 reports is found in Appendix D (Supplementary file).

\subsection{Study characteristics}

266 The 596 included studies were most commonly conducted in North America (74\%; Table 1). The

267 studies were published from 2000 to 2016, with $69 \%$ of studies published in the last 10 years

268 (2007 to 2016), 37\% published in the last 5 years (2012 to 2016), and 20\% published in the last 3

269 years (2014 to 2016). Figure 2 shows the publication of studies over time. The funding source

270 was public in $74 \%$, private or a mix of public and private in $7 \%$, no funding in $3 \%$, and not

271 reported in $17 \%$ of studies.

273 The context included cancer in 350 studies (59\%) or other chronic diseases in 295 studies (49\%);

27449 studies (8\%) examined patients with cancer and other types of chronic diseases. The five most

275 common cancer types (including prevention and risk factors) were breast (21\%), general/all

276 (cancer) types (13\%), colorectal (8\%), cervical (7\%), and lung (6\%). The five most common

277 chronic diseases (including prevention and risk factors) were cardiovascular (11\%), diabetes

278 (9\%), general/all (chronic disease) types (9\%), obesity/overweight (8\%), and other/unspecified

279 (8\%; Table 1).

280 The included studies reported using KT theories, models or frameworks to guide $627 \mathrm{KT}$

281 interventions to disseminate or implement 927 clinical interventions. The most frequent study 
design was experimental (50\%) followed by observational (19\%) and qualitative (18\%). The most common intervention settings were community only (35\%), outpatient clinic only (23\%), and multiple settings (i.e., at least two of: community, outpatient clinic, hospital, home, government, and long-term care; $21 \%$ ). The majority of the interventions targeted patients/general public (85\%), while $31 \%$ targeted healthcare professionals, and 23\% targeted organizations. Further, $30 \%$ of the KT interventions targeted more than one stakeholder group (i.e., at least two of: patients/general public, healthcare professionals, organizations, and financial/regulatory; Table 1).

\subsection{KT theory, model and framework characteristics}

We identified 159 different KT theories, models and frameworks. Appendix E (Supplementary

file) summarizes their key characteristics across the included studies, and organizes them by the stage of KT they were used for; the level of behavior change they targeted; the context (cancer, chronic disease, or both); whether they were used prospectively or retrospectively (i.e., after the study was completed); and the total number of studies in which they were used.

297 Overall, the three most frequently used theories, models or frameworks were Bandura's Social

298 Cognitive Theory (168 studies), Prochaska and DiClemente's Transtheoretical Model of Behavior

299 Change (141 studies) and Rosenstock's Health Belief Model (67 studies). In contrast, 139 (87\%)

300 of the theories, models and frameworks were used in five or fewer studies, with $95(60 \%)$ used

301 once. The majority (91\%) of theories, models and frameworks were reported to be used

302 prospectively. 
305 Most frequently, the 159 identified theories, models and frameworks were used to inform

306 planning/design (81\%), implementation (67\%) and evaluation (55\%) activities. The theories,

307 models and frameworks were less frequently used to inform dissemination (32\%) and

308 sustainability and/or scalability activities (23\%).

309

310 Twenty-six theories, models or frameworks were used across all four KT stages of

311 planning/design, implementation, evaluation, and sustainability/scalability; we call these 'full-

312 spectrum theories, models or frameworks'. This category includes theories, models and

313 frameworks that were used to inform the four KT stages either within a single study (as was the

314 case in 32 out of 421 studies that used a full-spectrum theory, model or framework) or across

315 different studies. Table 2 presents the 26 full-spectrum theories, models and frameworks and

316 organizes them by KT intervention target; level of behavior change; context (cancer, chronic

317 disease, or both); and stage of cancer/chronic disease care continuum. Additional details

318 concerning study design, setting and outcomes for the 26 full-spectrum theories, models and

319 frameworks are found in Appendix F (Supplementary file).

321 Of the 26 full-spectrum theories, models and frameworks, 18 were used prospectively, two were

322 used retrospectively, and six were used both prospectively and retrospectively in different studies.

323 As shown in Table 2, cancer control (with or without other chronic diseases) was the context for

32421 of the 26 full spectrum theories, models and frameworks; of these, 18 theories, models or

325 frameworks covered more than one stage of care, of which two covered the full cancer control

326 continuum (Action Research, and Rogers's Diffusion of Innovations Theory). Overall, the 21 full-

327 spectrum theories, models and frameworks used in cancer control were most often used in studies 
on cancer prevention, screening, treatment and survivorship. 'Other chronic diseases only' was the context for five of the full-spectrum theories, models and frameworks; of these, one theory, model or framework (Institute for Healthcare Improvement's Collaborative Model for Achieving

331 Breakthrough Improvement) covered more than one stage of care, and none covered the full

332 chronic disease control continuum. The five full-spectrum theories, models or frameworks used in 333 chronic disease care only were most often used in studies on prevention and treatment. Fifteen of 334 the 26 full-spectrum theories, models and frameworks were also used for dissemination, thus 335 covering the full KT continuum.

\subsection{Level of behavior change}

338 All 159 theories, models and frameworks were used for at least individual-level change, while

$33948 \%$ were used for organization-level, $33 \%$ for community-level, and $17 \%$ for system-level 340 change. Overall, $11 \%$ of the identified theories, models and frameworks addressed all four levels

341 of behavior change across the included studies. Of the 26 full-spectrum theories, models and

342 frameworks (used across the planning/design, implementation, evaluation, and

343 sustainability/scalability stages), 24 were used for organization-level change, 19 for community-

344 level change, and 11 for system-level change. Overall, 10 full-spectrum theories, models and 345 frameworks addressed all four levels of behavior change across the included studies (Table 2).

\section{4. DISCUSSION}

348 To our knowledge, this is the first comprehensive review of KT theories, models and frameworks

349 that have been used to guide dissemination or implementation of evidence-based interventions

350 targeted to prevention and/or management of cancer or other types of chronic diseases. We 
identified 596 studies (plus 47 companion reports) reporting on the use of 159 KT theories, models or frameworks used to inform $627 \mathrm{KT}$ interventions.

354 Of the 159 identified KT theories, models and frameworks, only three were used in more than $35510 \%$ of the included studies: Social Cognitive Theory, Transtheoretical Model of Behavior 356 Change, and Health Belief Model. These theories, models and frameworks have been developed

357 from different fields including education and psychology amongst others. This diversity

358 highlights the challenge for those interested in KT to conduct broad literature searches including 359 other disciplines to inform their work. Moreover, this also underscores the critical need to test the 360 application of these theories, models and frameworks that are developed in other fields in the KT 361 and healthcare contexts. For example, little is known on whether these theories, models and

362 frameworks can predict individual behavior change in healthcare (Table 2). In contrast, 139

$363(87 \%)$ theories, models and frameworks were used in less than $1 \%$ of the included studies (i.e.,

364 five or fewer studies), with 95 (60\%) used once. Our findings highlight the significant number of

365 KT theories, models and frameworks currently available and the limited evidence-base describing 366 their use in practice.

368 The 159 identified theories, models and frameworks were most commonly used to inform 369 planning/design, implementation, and evaluation activities, and least commonly used to inform 370 dissemination and sustainability and/or scalability activities. Twenty-six were used across the full 371 KT spectrum, from planning/design to sustainability/scalability. While we used the stages of the

372 Knowledge-to-Action cycle in a linear approach to frame this review, this is not how the cycle 373 was intended to be used. It is a cycle and as such it should be used in an iterative fashion. 
374 However, this is an element that could not be explicitly captured from the theories, models and

375 frameworks - namely, how they approach the iterative nature of KT work. Although the

376 Knowledge-to-Action cycle is one of the most commonly-used process models, there are others

377 we could have used to structure our findings.

378

379 With most of the $627 \mathrm{KT}$ interventions targeted to individuals (e.g., patients/general public or

380 healthcare professionals), it is not surprising that all of the identified theories, models and

381 frameworks covered at least individual-level behavior change, while only a few covered system-

382 level change. Given the complexity of healthcare system change, this is a limitation of these

383 theories, models and frameworks. Typically in KT work, there are multiple behaviors being

384 changed among multiple actors (patients, clinicians, healthcare managers, policy makers) and

385 across various settings. For KT science to advance and inform KT practice, it is critical to have

386 valid theories, models and frameworks that address system-level change.

388 Compared to a previously published review by Tabak and colleagues [14] describing 61 theories, models and frameworks, our scoping review included more than double the number of theories, models and frameworks. Despite including the names of all 61 theories, models and frameworks in our search, and similarly focusing on all levels of behavior change, we did not expect all of

392 them to be included in our review, considering our narrower practice versus research focus and

393 the focus on cancer and other types of chronic diseases. A recent citation network analysis of 63

394 KT theories, models and frameworks found that while some were highly cited (such as the

395 Knowledge-to-Action Framework), most were rarely cited in the literature from 1985 to 2012

396 [17]. Another citation analysis by Field and colleagues explored use of the Knowledge-to-Action 
397 Framework in the literature from 2006 to 2013 [32]. Out of 146 identified studies, the authors

398 found that 101 studies either cited the model without explanation or used it to guide the project in

399 a 'general, non-specified way', while only 10 studies used the model to inform 'the design,

400 delivery, and evaluation of implementation activities' [32]. Similarly, our scoping review

401 identified a significant number of KT theories, models or frameworks with a limited evidence-

402 base describing their use in practice. As such, the KT field would benefit from better reporting

403 [33], including a more detailed description by study authors of how they used the theory, model

404 or framework, if one was selected. However, with over 150 identified KT theories, models or

405 frameworks to choose from, it can be difficult to know which one(s) would be an appropriate

406 choice - especially when some of the theories, models and frameworks identified in this review

407 included, were informed by, or modified other existing theories, models or frameworks. It would

408 also be useful to conduct a future study to understand how individuals in the field select what

409 theory, model and/or framework to use to inform their work and why ones such as the

410 Knowledge-to-Action cycle are cited more frequently than others.

411

412 Given the large number of KT theories, models and frameworks available, our summary tables

413 can be searched for theories, models or frameworks depending on the details of the dissemination

414 or implementation project. For instance, an end user might consider whether they are looking for

415 a theory or a model or a framework to inform their entire organizational activities, their individual

416 projects, or both. Other criteria to consider might include theories, models or frameworks that

417 cover multiple steps in the KT process (e.g., full-spectrum theories, models and frameworks),

418 those that have been used across the cancer/chronic disease control continuum, as well as those

419 that were reported as being used prospectively. 
421 A decision support tool to help end users identify an appropriate KT theory, model or framework to inform their work would therefore be highly beneficial. While a similar tool does exist for KT researchers [34], the tool is not based on a comprehensive systematic or scoping review approach. Therefore, we intend to use the findings of our scoping review to inform the development of a comprehensive tool to help end users identify an appropriate theory to inform their KT activities. Such a tool has the potential to benefit the healthcare system by helping frontline users develop better interventions and achieve greater success when implementing evidence into practice. It also seems clear that while the KT field is changing rapidly, as evidenced by the ever-increasing number of theories, models and frameworks being published in recent years, there is little evidence that any consolidation of a few theories, models or frameworks is taking place [35].

431 Such efforts could help both guide the field and aid implementation practitioners and researchers in translating the lessons learned from science into more broadly implementing evidence-based interventions in cancer and chronic disease prevention and control.

435 There are limitations to our scoping review worth noting. First, there is potential for 436 misclassification of theories, models and frameworks. Different terms (theory versus model 437 versus framework) were used by study authors when describing the theories, models and 438 frameworks both across and within their own studies, which highlights that some were 439 misclassified by study authors. The main purpose for using the BeHEMoTh approach to identify 440 theories, models and frameworks for the search strategy was to account for these differences.

441 Thus, we abstracted and reported the terms as used by the study authors, while additional terms 
442 that were used (e.g., approach) were abstracted as 'other'. Consistent terminology is important

443 because theories, models and frameworks serve different purposes (e.g., Nilsen's taxonomy) [5].

444

445 Second, we limited our literature search to English language articles and excluded book chapters

446 [36]. As such, it is not clear how many non-English articles may have been excluded. Despite

447 this, we identified almost 600 studies, making it the largest review of KT theories, models and

448 frameworks to our knowledge. Further, we included studies conducted in different continents,

449 suggesting applicability of the findings for other countries. Third, we focused on the prevention

450 and/or management of cancer and other chronic diseases. However, this was inclusive of chronic

451 mental illness as well as risk factors for cancer and chronic disease. Therefore, the scope of our

452 review was quite broad and the findings are applicable to a wide range of health areas including

453 public health. Fourth, we did not assess the limitations to the different theories, models and

454 frameworks, such as whether or not it is appropriate for a certain context or is lacking validity

455 evidence [3]. For instance, Prochaska and DiClemente's Transtheoretical Model of Behavior

456 Change is an example of a widely-used theory, model or framework that lacks supporting

457 evidence of effectiveness from systematic reviews [37]. The use of an operational definition of a

458 theory, a model and a framework could have affected the number identified and included in our

459 review. As such, we chose to code the theories, models and frameworks based on how the study

460 authors reported using them to inform their work, and not based on the intended use according to

461 the developer or the constructs present in the theory, model or framework. Whether or not the

462 study authors correctly selected and applied the theory, model or framework was beyond the

463 scope of this review and highlights something that could be explored in a future project. Further,

464 we chose to report on the number of times each theory, model and framework was used in 
465 practice to help map the current literature; however, we would strongly caution against using the

466 frequency of use as an indicator of quality. Assessment of the quality of various theories, models

467 and frameworks was out of scope and would require separate search strategies of each of the

468 included theories, models and frameworks to identify evidence of their validity.

469

470 Our scoping review found a significant number of KT theories, models and frameworks with a

471 limited evidence-base describing their use in practice. A comprehensive tool to help end users

472 identify an appropriate theory, model or framework to inform their KT activities would be

473 beneficial. Subsequent work will aim to develop and test a decision support tool for end users. 


\section{LIST OF ABBREVIATIONS}

$475 \mathrm{KT}=$ Knowledge translation

476

477 FIGURES

478 Figure 1. Study flow diagram

479 Figure 2. Number of studies reporting on use of KT theories/models/frameworks over time 480

481 ADDITIONAL FILES

482 File name: Supplementary file .pdf

483 Title: Appendices A-F

484 Description: Supplementary file with appendices A-F

485

486 DECLARATIONS

487 Ethics Approval and Consent to Participate

488 Not applicable

489 Consent for Publication

$490 \quad$ Not applicable

$491 \quad$ Availability of Data and Material

492 Not applicable

493 Competing Interests

494 Jessie McGowan, Andrea Tricco and Sharon Straus are on the editorial board for the Journal, but

495 were not involved with the peer review process or decision to publish. All other authors declare

496 that they have no competing interests. 
$497 \quad$ Funding

498 This work was funded by the Canadian Partnership Against Cancer. Strifler is funded by a

499 Canadian Institutes of Health Research Banting Doctoral Award. Tricco is funded by a Tier 2

500 Canada Research Chair in Knowledge Synthesis. Straus is funded by a Tier 1 Canada Research

501 Chair in Knowledge Translation and the Mary Trimmer Chair in Geriatric Medicine.

502 Author Contributions

503 Study concept and design: Strifler, Tricco, Straus. Developed the search strategy: McGowan.

504 Data acquisition, cleaning, or interpretation: Strifler, Cardoso, Cogo, Nincic, Khan, Scott,

505 Ghassemi, MacDonald, Lai, Treister, Tricco, Straus. Drafted the manuscript: Strifler, Straus.

506 Provided input and revised the manuscript: Strifler, Cardoso, McGowan, Cogo, Nincic, Khan,

507 Scott, Ghassemi, MacDonald, Lai, Treister, Tricco, Straus. Obtained funding: Straus. Supervised

508 the study: Strifler, Straus. All authors read and approved the final manuscript.

509 Acknowledgements

510 The authors thank Louise Zitzelsberger and Jon Kerner (Canadian Partnership Against Cancer)

511 for their involvement as knowledge users throughout the review process and for reviewing the

512 manuscript; and Annemarie Edwards (Canadian Partnership Against Cancer) for reviewing the

513 manuscript. The authors also thank John Ivory for screening some of the articles, Alissa Epworth

514 for locating full-text articles, and Inthuja Selvaratnam for formatting the manuscript. 
[1] Straus SE, Tetroe JM, Graham ID. Knowledge translation is the use of knowledge in health care decision making. Journal of clinical epidemiology. 2011;64:6-10.

[2] Eccles M, Grimshaw J, Walker A, Johnston M, Pitts N. Changing the behavior of healthcare professionals: the use of theory in promoting the uptake of research findings. Journal of clinical epidemiology. 2005;58:107-12.

[3] Davidoff F, Dixon-Woods M, Leviton L, Michie S. Demystifying theory and its use in improvement. BMJ Qual Saf. 2015;24:228-38.

[4] Davies P, Walker AE, Grimshaw JM. A systematic review of the use of theory in the design of guideline dissemination and implementation strategies and interpretation of the results of rigorous evaluations. Implement Sci. 2010;5:14.

[5] Nilsen P. Making sense of implementation theories, models and frameworks. Implement Sci. 2015;10:53.

[6] Rogers E. Diffusion and Innovations. Glencoe: The Free Press(1976)," New Product Adoption and Diffusion," The Journal of Consumer Research. 1962;2:290-301.

530 [7] Bandura A. Social Foundations of Thought and Action: a Social Cognitive Theory Prentice-

531 Hall: New York, 1986.

532 [8] May C, Finch T. Implementing, embedding, and integrating practices: an outline of

533 normalization process theory. Sociology. 2009;43:535-54.

534 [9] Graham ID, Logan J, Harrison MB, Straus SE, Tetroe J, Caswell W, et al. Lost in knowledge 535 translation: time for a map? J Contin Educ Health Prof. 2006;26:13-24.

536 [10] Meyers DC, Durlak JA, Wandersman A. The quality implementation framework: A synthesis 537 of critical steps in the implementation process. American journal of community psychology.

538 2012;50:462-80.

539 [11] Michie S, Johnston M, Abraham C, Lawton R, Parker D, Walker A. Making psychological

540 theory useful for implementing evidence based practice: a consensus approach. Quality and safety 541 in health care. 2005;14:26-33.

542 [12] Damschroder LJ, Aron DC, Keith RE, Kirsh SR, Alexander JA, Lowery JC. Fostering 543 implementation of health services research findings into practice: a consolidated framework for 544 advancing implementation science. Implement Sci. 2009;4:50.

545 [13] Glasgow RE, Vogt TM, Boles SM. Evaluating the public health impact of health promotion 546 interventions: the RE-AIM framework. American journal of public health. 1999;89:1322-7.

547 [14] Tabak RG, Khoong EC, Chambers DA, Brownson RC. Bridging research and practice: 548 models for dissemination and implementation research. American journal of preventive medicine. 549 2012;43:337-50.

550 [15] Straus S, Tetroe J, Graham ID. Knowledge translation in health care: moving from evidence 551 to practice. 2nd ed. Oxford, UK: John Wiley \& Sons; 2013. 
552 [16] Colquhoun HL, Brehaut JC, Sales A, Ivers N, Grimshaw J, Michie S, et al. A systematic

553 review of the use of theory in randomized controlled trials of audit and feedback. Implement Sci.

554 2013;8:66.

555 [17] Skolarus TA, Lehmann T, Tabak RG, Harris J, Lecy J, Sales AE. Assessing citation

556 networks for dissemination and implementation research frameworks. Implement Sci.

$557 \quad 2017 ; 12: 97$.

558 [18] Arksey H, O'Malley L. Scoping studies: Towards a Methodological Framework.

559 International journal of social research methodology. 2005;8:19-32.

560 [19] Peters MDJ, Godfrey C, McInerney P, Soares CB, Khalil H, Parker D. Methodology for JBI

561 Scoping reviews. The Joanna Briggs' Institute Reviewers' Manual 2015. 2015. Accessed October

562 2016. Available from http://joannabriggs.org/assets/docs/sumari/Reviewers-

563 Manual_Methodology-for-JBI-Scoping-Reviews_2015_v2.pdf

564 [20] Canadian Institutes of Health Research. Knowledge Translation. 2016. Accessed. Available

565 from http://www.cihr-irsc.gc.ca/e/29418.html

566 [21] National Implementation Research Network. Implementation Defined. 2018. Accessed

567 March 2018. Available from http://nirn.fpg.unc.edu/learn-implementation/implementation-

568 defined

569 [22] Canadian Institutes of Health Research. Integrated knowledge translation (iKT). 2018.

570 Accessed March 2018. Available from http://www.cihr-irsc.gc.ca/e/45321.html\#a3

571 [23] Moher D, Liberati A, Tetzlaff J, Altman DG. Preferred reporting items for systematic

572 reviews and meta-analyses: the PRISMA statement. Annals of internal medicine. 2009;151:264-9.

573 [24] Booth A, Carroll C. Systematic searching for theory to inform systematic reviews: is it

574 feasible? Is it desirable? Health Information \& Libraries Journal. 2015;32:220-35.

575 [25] McGowan J, Sampson M, Salzwedel DM, Cogo E, Foerster V, Lefebvre C. PRESS Peer

576 Review of Electronic Search Strategies: 2015 Guideline Statement. Journal of clinical

577 epidemiology. 2016;75:40-6.

578 [26] Canadian Agency for Drugs Technologies in Health. Grey Matters: a practical tool for search 579 health-related grey literature. CADTH Information Services. 2016.

580 [27] Knowledge Translation Program. Synthesi.SR. 2014. Accessed September 2016. Available

581 from http://www.breakthroughkt.ca/login.php

582 [28] Effective Practice and Organisation of Care (EPOC). EPOC Taxonomy. 2015. Accessed

583 September 2017. Available from https://epoc.cochrane.org/epoc-taxonomy

584 [29] Sallis JF, Owen N, Fisher E. Ecological models of health behavior. Health behavior: theory, 585 research, and practice 5th ed San Francisco: Jossey-Bass. 2015:43-64.

586 [30] Cochrane Consumers and Communication. Consumers and Communication Group resources 587 for authors. 2017. Accessed August 2017. Available from http://cccrg.cochrane.org/author-

588 resources

589 [31] Tricco AC, Lillie E, Zarin W, O'Brien K, Colquhoun H, Kastner M, et al. A scoping review 590 on the conduct and reporting of scoping reviews. BMC medical research methodology.

$591 \quad 2016 ; 16: 15$. 
592 [32] Field B, Booth A, Ilott I, Gerrish K. Using the Knowledge to Action Framework in practice:

593 a citation analysis and systematic review. Implement Sci. 2014;9:172.

594 [33] The Equator Network. Enhancing the QUAlity and Transparency Of health Research.

595 Accessed March 2018. Available from http://www.equator-network.org/

596 [34] The Center for Research in Implementation Science and Prevention. Dissemination \& 597 Implementation Models in Health Research \&Practice. Accessed March 2018. Available from

598 http://www.dissemination-implementation.org/select.aspx

599 [35] Brehaut JC, Eva KW. Building theories of knowledge translation interventions: use the entire 600 menu of constructs. Implement Sci. 2012;7:114.

601 [36] Morrison A, Polisena J, Husereau D, Moulton K, Clark M, Fiander M, et al. The effect of 602 English-language restriction on systematic review-based meta-analyses: a systematic review of 603 empirical studies. International journal of technology assessment in health care. 2012;28:138-44.

604 [37] West R. Time for a change: putting the Transtheoretical (Stages of Change) Model to rest. 605 Addiction (Abingdon, England). 2005;100:1036-9. 
1 TABLES

2

3

Table 1. Study Characteristics

\begin{tabular}{rcc}
\hline & $\begin{array}{c}\text { No. of studies } \\
(\mathbf{n = 5 9 6})\end{array}$ & \% of studies \\
\hline Year of publication & & \\
$2007-2016$ & 412 & 69 \\
$2012-2016$ & 220 & 37 \\
\cline { 2 - 3 } $2014-2016$ & 121 & 20 \\
\hline
\end{tabular}

\section{Geographic region}

\begin{tabular}{|c|c|c|}
\hline & & \\
\hline North America & 438 & 73 \\
\hline Europe & 72 & 12 \\
\hline Australia \& New Zealand & 40 & 7 \\
\hline Asia & 31 & 5 \\
\hline Africa & 5 & 1 \\
\hline Intercontinental & 4 & 1 \\
\hline Central \& South America & 6 & 1 \\
\hline Context $^{\mathrm{f}}$ & & \\
\hline Cancer $^{*}$ & 350 & 59 \\
\hline Breast & 125 & 21 \\
\hline General/all types & 79 & 13 \\
\hline Colorectal & 50 & 8 \\
\hline Cervical & 44 & 7 \\
\hline Lung & 33 & 6 \\
\hline Chronic disease $^{\mathrm{Y}}$ & 295 & 49 \\
\hline Cardiovascular disease & 68 & 11 \\
\hline Diabetes & 56 & 9 \\
\hline General/all types & 53 & 9 \\
\hline Obesity/overweight & 50 & 8 \\
\hline Other/unspecified & 45 & 8 \\
\hline & $\begin{array}{c}\text { No. of } \\
\text { interventions } \\
(n=627)\end{array}$ & $\begin{array}{c}\% \text { of } \\
\text { interventions }\end{array}$ \\
\hline
\end{tabular}

\section{Intervention target*}

Patients/general public

Healthcare professionals

Organization

Financial/regulatory

All 4 target categories

$>1$ target categories

\begin{tabular}{cc}
528 & 84 \\
\hline 206 & 33 \\
\hline 142 & 23 \\
\hline 34 & 5 \\
\hline 6 & 1 \\
\hline 192 & 31
\end{tabular}

\section{Intervention setting*}

\begin{tabular}{rcc} 
Community & 228 & 36 \\
\cline { 2 - 3 } Clinic (outpatients) & 149 & 24 \\
\cline { 2 - 3 } Multiple settings & 141 & 22 \\
\cline { 2 - 3 } Home & 69 & 11 \\
\cline { 2 - 3 }
\end{tabular}




\begin{tabular}{rcc}
\cline { 2 - 3 } Hospital (inpatients) & 26 & 4 \\
\cline { 2 - 3 } Not reported & 19 & 3 \\
\cline { 2 - 3 } Government & 4 & 1 \\
\cline { 2 - 3 } Long-term care & 1 & 0
\end{tabular}

\section{Study design*}

\begin{tabular}{rcc} 
Experimental & 302 & 48 \\
\cline { 2 - 3 } Observational & 128 & 20 \\
\cline { 2 - 3 } Qualitative & 116 & 18 \\
\cline { 2 - 3 } multi-methods & 34 & 5 \\
\cline { 2 - 3 } i-experimental & 29 & 5 \\
\cline { 2 - 3 } Unclear & 18 & 3
\end{tabular}

*Multiple health context, interventions, settings and study designs reported per study; ${ }^{\mathrm{F}}$ Most common reported;

${ }^{¥}$ Includes risk factors 


\begin{tabular}{|c|c|c|c|c|c|c|c|c|c|c|c|c|c|c|c|c|c|}
\hline \multirow[b]{2}{*}{ Name of KT theory, model or framework } & \multicolumn{4}{|c|}{$\mathrm{KT}$ intervention target } & \multicolumn{4}{|c|}{ Behavior change level } & \multicolumn{3}{|c|}{ Continuum of care } & & & & & \multirow[b]{2}{*}{ Context } & \multirow[b]{2}{*}{$\begin{array}{l}\text { \# of } \\
\text { studies* }\end{array}$} \\
\hline & 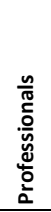 & 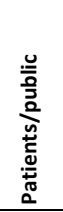 & 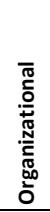 & 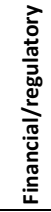 & 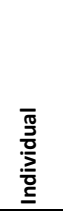 & 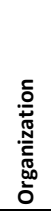 & 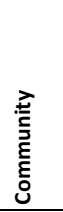 & 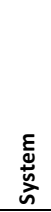 & 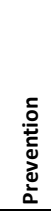 & 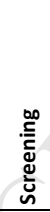 & 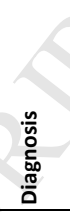 & 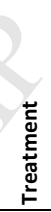 & 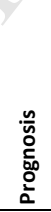 & 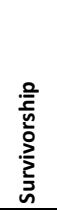 & 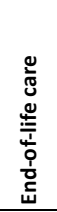 & & \\
\hline Action Research & 22 & 31 & 21 & 3 & 41 & 21 & 15 & 1 & 14 & 13 & 4 & 22 & 3 & 8 & 5 & Both & 42 \\
\hline $\begin{array}{l}\text { CAN-IMPLEMENT (Canadian Guideline Adaption Study } \\
\text { Group) }\end{array}$ & 8 & 1 & 7 & 0 & 4 & 4 & 0 & 1 & 0 & 0 & 2 & 4 & 2 & 2 & 0 & Cancer & 4 \\
\hline $\begin{array}{l}\text { Collaborative Model for Achieving Breakthrough } \\
\text { Improvement (Institute for Healthcare Improvement) }\end{array}$ & 6 & 5 & 4 & 2 & 4 & 4 & 2 & 2 & 1 & 1 & 1 & 3 & 0 & 0 & 2 & $\begin{array}{l}\text { Chronic } \\
\text { Disease }\end{array}$ & 3 \\
\hline Community Connection Model (Liddy et al.) & 1 & 1 & 1 & 0 & 1 & 1 & 1 & 0 & 0 & 0 & 0 & 1 & 0 & 0 & 0 & $\begin{array}{l}\text { Chronic } \\
\text { Disease }\end{array}$ & 1 \\
\hline $\begin{array}{l}\text { Consolidated Framework for Implementation } \\
\text { Research (CFIR) (Damschroder) }\end{array}$ & 5 & 3 & 4 & 0 & 6 & 4 & 1 & 0 & 3 & 1 & 0 & 5 & 0 & 1 & 0 & Both & 7 \\
\hline $\begin{array}{l}\text { Diffusion of Health Promotion Innovations (Oldenburg } \\
\text { et al.) }\end{array}$ & 1 & 0 & 1 & 0 & 1 & 1 & 0 & 0 & 1 & 0 & 0 & 0 & 0 & 0 & 0 & $\begin{array}{l}\text { Chronic } \\
\text { Disease }\end{array}$ & 1 \\
\hline Diffusion of Innovations (Rogers) & 26 & 20 & 15 & 6 & 37 & 17 & 13 & 7 & 22 & 10 & 1 & 12 & 2 & 2 & 1 & Both & 37 \\
\hline Interactive Systems Framework (Wandersman) & 5 & 2 & 1 & 0 & 1 & 11 & 0 & 0 & 1 & 1 & 0 & 1 & 0 & 1 & 0 & Cancer & 1 \\
\hline Interorganizational Relations Theory & 1 & 1 & 1 & 0 & 1 & 1 & 1 & 0 & 1 & 0 & 0 & 0 & 0 & 0 & 0 & Cancer & 1 \\
\hline Knowledge-to-Action (KTA) (Graham et al.) & 3 & 2 & 2 & 0 & 4 & 2 & 0 & 0 & 0 & 0 & 1 & 4 & 1 & 1 & 0 & Both & 4 \\
\hline LEAN Transformation Process & 2 & 1 & 2 & 0 & 2 & 2 & 0 & 0 & 0 & 0 & 0 & 2 & 0 & 0 & 0 & Cancer & 2 \\
\hline $\begin{array}{l}\text { Model for Accelerating Improvement (Associates in } \\
\text { Process Improvement) }\end{array}$ & 4 & 2 & 3 & 0 & 4 & 3 & 1 & 1 & 0 & 1 & 0 & 3 & 0 & 0 & 0 & Both & 4 \\
\hline Plan-Do-Study-Act (PDSA) Cycles (Deming) & 22 & 17 & 21 & 12 & 16 & 12 & 2 & 2 & 4 & 3 & 1 & 14 & 0 & 0 & 1 & Both & 16 \\
\hline $\begin{array}{l}\text { Precaution Adoption Process Model (PAPM) } \\
\text { (Weinstein \& Sandman) }\end{array}$ & 0 & 8 & 0 & 0 & 8 & 0 & 0 & 0 & 5 & 3 & 0 & 0 & 0 & 0 & 0 & Both & 8 \\
\hline PRECEDE-PROCEED (Green) & 6 & 18 & 4 & 0 & 17 & 5 & 4 & 1 & 5 & 9 & 0 & 4 & 0 & 0 & 0 & Both & 17 \\
\hline $\begin{array}{l}\text { Quality Implementation Framework (Meyers, Durlak \& } \\
\text { Wandersman) }\end{array}$ & 1 & 0 & 1 & 1 & 1 & 1 & 0 & 0 & 0 & 0 & 0 & 1 & 0 & 1 & 0 & Cancer & 1 \\
\hline $\begin{array}{l}\text { Reach Effectiveness Adoption Implementation } \\
\text { Maintenance (RE-AIM) (Glasgow et al.) }\end{array}$ & 13 & 20 & 7 & 3 & 18 & 4 & 3 & 0 & 15 & 4 & 0 & 9 & 1 & 2 & 2 & Both & 23 \\
\hline Self-Regulation Theory & 0 & 12 & 0 & 0 & 14 & 0 & 1 & 0 & 6 & 1 & 0 & 6 & 1 & 1 & 0 & Both & 14 \\
\hline Social Cognitive Theory (Bandura) & 16 & 135 & 9 & 4 & 168 & 19 & 20 & 5 & 79 & 15 & 1 & 57 & 11 & 27 & 0 & Both & 168 \\
\hline
\end{tabular}




\begin{tabular}{llllllllllllllllllll}
\hline Social Ecology Model for Health Promotion (Stokols) & 0 & 2 & 0 & 0 & 4 & 1 & 2 & 0 & 3 & 1 & 0 & 0 & 0 & 0 & Both & 4 \\
\hline Social Learning Theory (Bandura) & 2 & 23 & 2 & 0 & 23 & 2 & 2 & 1 & 11 & 5 & 0 & 8 & 0 & 2 & 0 & Both & 23 \\
\hline Social Marketing Framework & 2 & 10 & 2 & 0 & 10 & 2 & 5 & 0 & 5 & 3 & 0 & 3 & 0 & 0 & 0 & Both & 10 \\
\hline Stage Theory of Organizational Change & 1 & 1 & 1 & 0 & 1 & 1 & 1 & 0 & 1 & 0 & 0 & 0 & 0 & 0 & 0 & Cancer & 1 \\
\hline Three-World View Model (Peek) & 1 & 1 & 1 & 1 & 1 & 1 & 1 & 0 & 0 & 0 & 0 & 1 & 0 & 0 & 0 & $\begin{array}{l}\text { Chronic } \\
\text { Disease }\end{array}$ & 1 \\
\hline $\begin{array}{l}\text { Transtheoretical Model of Behaviour Change } \\
\text { (Prochaska \& DiClemente) }\end{array}$ & 17 & 142 & 10 & 4 & 142 & 11 & 10 & 3 & 88 & 31 & 0 & 22 & 0 & 17 & 0 & Both & 141 \\
\hline $\begin{array}{l}\text { Western Australia Health Network Policy } \\
\text { Development and Implementation Cycle }\end{array}$ & 4 & 3 & 4 & 4 & 1 & 1 & 1 & 1 & 0 & 0 & 0 & 1 & 0 & 0 & 0 & $\begin{array}{l}\text { Chronic } \\
\text { Disease }\end{array}$ & 1 \\
\hline \begin{tabular}{l} 
Note: *References to studies can be found in the supplementary file \\
\hline
\end{tabular}
\end{tabular}



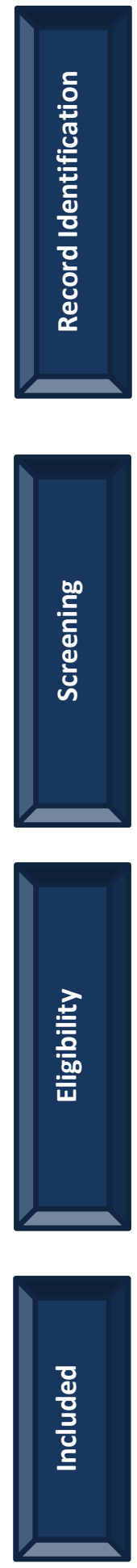

- BeHEMoTh/Main search $(n=1664)$

- Named search ( $n=1989)$

- Forward citation search $(n=235)$

- Grey literature search ( $n=977)$

- Systematic reviews screening $(n=319)$

- Includes searching for companion reports $(n=4)$

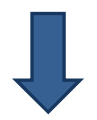

\section{Records after duplicates removed} $(n=4598)$

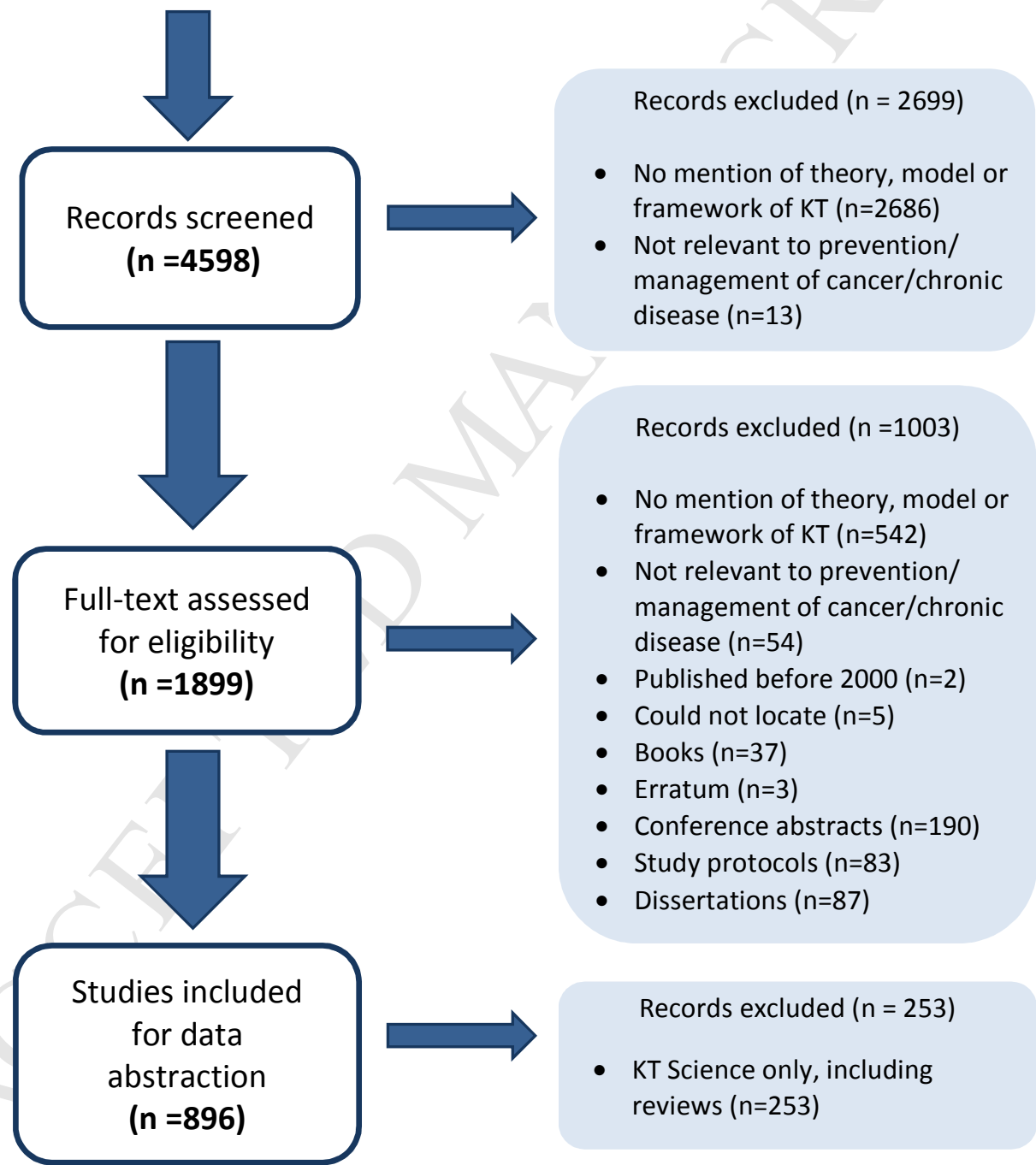

596 studies plus 47 companion reports included for a total of $\mathrm{N}=643$ studies 
Figure 2. Number of studies reporting on use of KT theories/models/frameworks over time

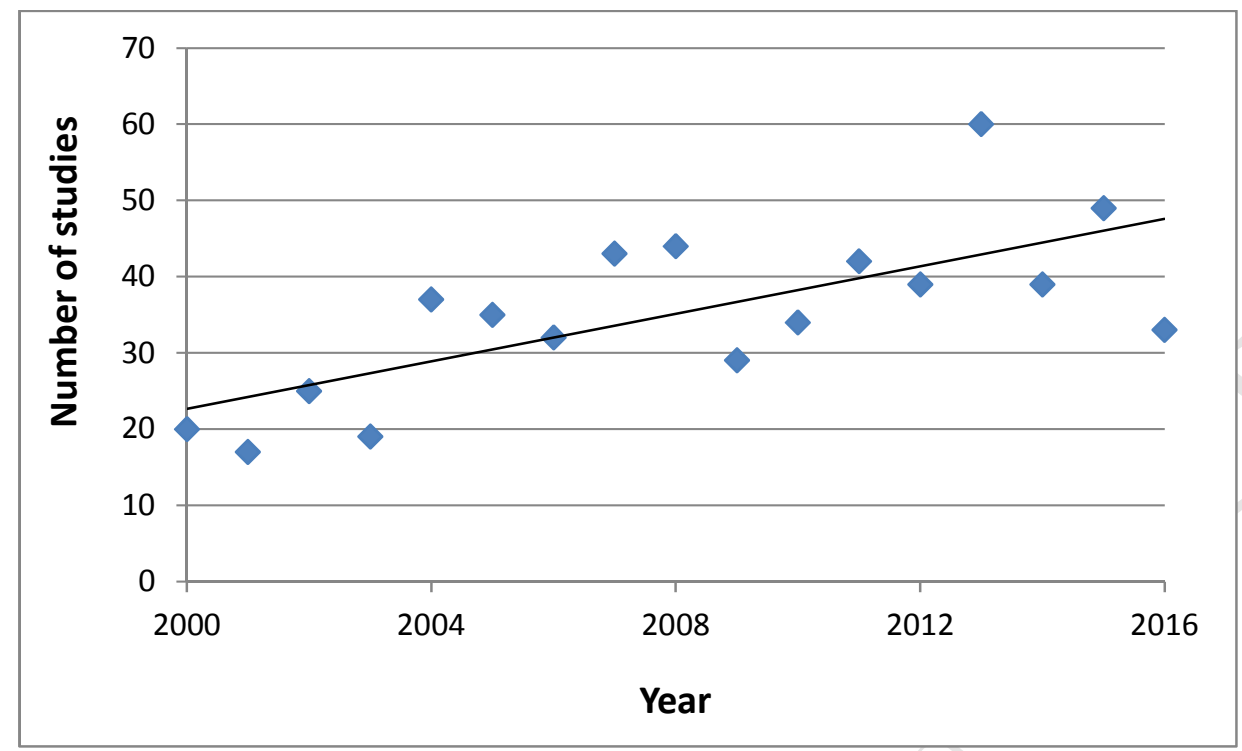




\section{What is new?}

\section{Key findings}

- We employed a new, iterative approach called the BeHEMoTh (Behavior of interest; Health context; Exclusions; Models or Theories) framework to inform our search protocol and to build a list of names of knowledge translation (KT) theories, models and frameworks.

- Our scoping review identified 596 studies reporting on the use of 159 KT theories, models or frameworks. A majority of the theories, models and frameworks were used in less than $1 \%$ (i.e., five or fewer) of the included studies, with many used once.

\section{What this adds to what was known?}

- To our knowledge, this is the first comprehensive review of KT theories, models and frameworks that have been used to guide dissemination or implementation of evidence-based interventions targeted to prevention and/or management of cancer or other types of chronic diseases.

\section{What is the implication and what should change now?}

- We found a significant number of KT theories, models and frameworks with a limited evidence base describing their use in practice. Our results suggest that a decision support tool to help end users identify an appropriate theory, model or framework to inform their KT activities would be beneficial. 